\title{
MAPPIng Algorithm, Simulating LidAR AND SONARS ON MOBILE ROBOTS.
}

\author{
Aleksei Plotnikov \& Valentin Pryanichnikov
}
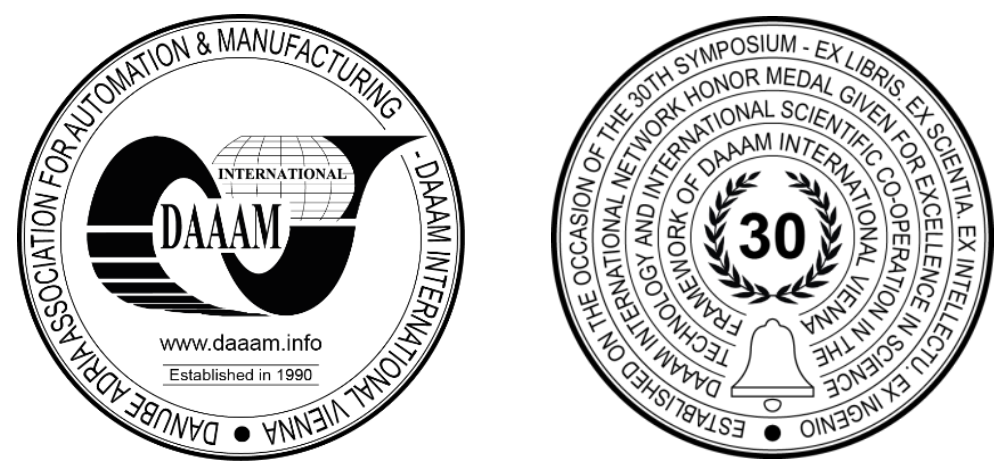

This Publication has to be referred as: Plotnikov, A[leksei] \& Pryanichnikov, V[alentin] (2020). Mapping Algorithm, Simulating Lidar and Sonars on Mobile Robots, Proceedings of the 31st DAAAM International Symposium, pp.09860993, B. Katalinic (Ed.), Published by DAAAM International, ISBN 978-3-902734-29-7, ISSN 1726-9679, Vienna, Austria

DOI: $10.2507 / 31$ st.daaam.proceedings. 136

\begin{abstract}
The paper proposes an algorithm for constructing a local map of the operational space of mobile service robots, based on logical analysis and comparison of readings from a small number of ultrasonic rangefinders (sonars). The described algorithm was tested in a virtual environment that simulates the robot's movement and the operation of sonar. The best results for the local mapping algorithm were found when placing sensors of 4 pairs on the sides of the robot so that for each pair the axes of the beam pattern would be deployed at a certain angle towards each other. To confirm the functionality of the sensor layout at work, an experiment was conducted to control a virtual robot in a test environment. Testing has shown that operators successfully identify walls, doorways, and obstacles while controlling the virtual robot. Experiments to find out the real directional pattern of HY-SRF05 sonar were conducted. And given thoughts on combining readings from sonar and LIDAR sensors.
\end{abstract}

Keywords: service mobile robots; mapping algorithms; sonar and lidar data processing.

\section{Introduction}

Currently, in robotics, more and more attention is paid to applications of mobile service robots (MSR) in various fields of activity. The use of MSR allows a person to perform various tasks, such as supervisory manipulation of objects or observation, without the need for the actual presence. As part of the joint work of several organizations (KIAM, INET RSUH, MSTU "Stankin" and others), service robots of the Amur series are being developed and implemented. These robots are intended for use in the network of laboratories and robotariums under the Intelligent robotics project, in particular, the AMUR-307 robot (Fig. 1), which continues the line of previously created training robots of this series [1],[2].[3].

During the creation of the mobile robot navigation algorithm, to ensure safety requirements, attention should be paid to the algorithm of detection and obstacle avoidance. Research and development of various SLAM algorithms have been successfully conducted for a long time. Developments in the ROS framework should be noted, as well as earlier algorithms for detecting and avoiding obstacles based on sonar data [4], [5], [6] or LIDAR data [7]. 


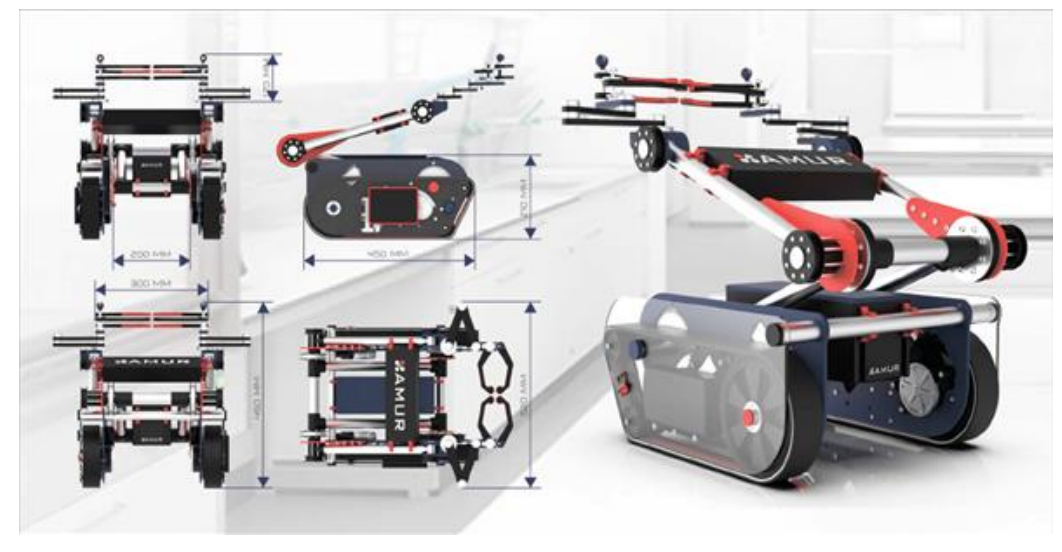

Fig. 1. AMUR-307 robot

Lidar makes it relatively easy to build a map of the robot's immediate environment. When using LIDAR on service mobile robot, the following difficulties arise:

- The cost of LIDAR exceeds the cost of other sensors by dozens of times.

- LIDAR used for mobile robots (Fig .2) have a lot of moving parts, which shortens time before failure during continuous operation and leads to increased maintenance costs.

- LIDAR have problems with transparent obstacles detection [8].

- When using LIDAR on a robot with manipulation devices, such as the AMUR-307, attention must be paid to blind spots. Blind spots formed due to the design features of the service robot can cover more than half of the LIDAR scanning area.

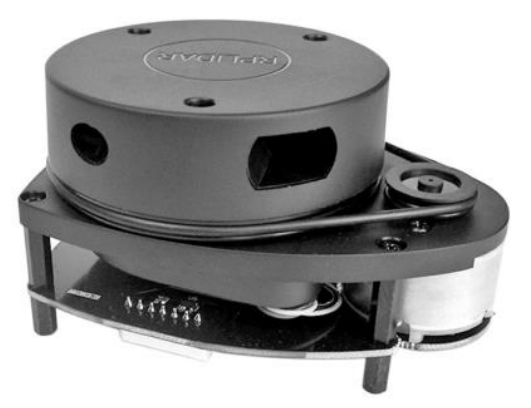

Fig. 2. Example of a mobile robot LIDAR

Sonars measure distances quite accurately, but have a very low angular resolution. At the same time, their low cost and simplicity give significant advantages over lidars. Based on the above, an algorithm was developed for determining obstacles in the nearest operating space of the robot based on the indication of a set of sonars. There are several works on sonar obstacle detection for mobile robots. There is proposition to use the signal coherence of ultrasonic waves with multichannel sonar signal processing [9], or rapid convergence of sonar and binocular stereovision sensor distance information [10].

\section{Description of the algorithm}

The following assumptions were made when developing algorithm:

- The robot operates on a two-dimensional space, where the position of the robot and obstacles are represented in the world coordinate system (WCS), Fig. 3.

- The area of operation of the sonar is taken as the sector, that originates at the point (spots), where the sensor is attached to the robot.

The mapping algorithm works in two-dimensional space, which corresponds to most tasks of robot navigation. At the preparatory stage, the following data must be set (Fig. 3, 4):

1. Cordinates $\mathrm{X}_{\mathrm{sR}}, \mathrm{Y}_{\mathrm{sR}}$ of the sensor location, direction of the sensor $\operatorname{Dir}_{\mathrm{sR}}$ in the robot coordinate system (CRS).

2. Sensor characteristics: maximum $\mathrm{R}_{\max }$ and minimum $\mathrm{R}_{\min }$ range of the sensor distance measuring, maximum scanning angle of the sensor cone $\mathrm{A}_{\text {cone }}$.

3. The maximum number of points formed inside the cone according to the sensor reading $\mathrm{N}$.

4. The radius around the point, that does not allow creating a point inside itself $R_{\text {exc }}$. 


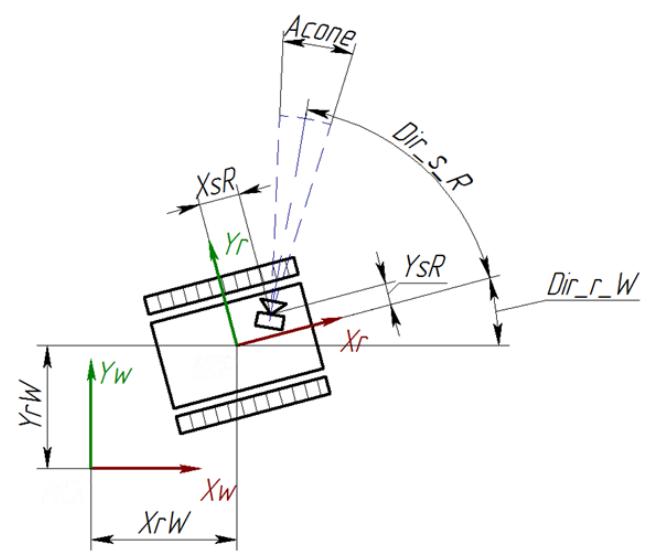

Fig. 3. World coordinate system (WCS) and robot coordinate system (RCS)

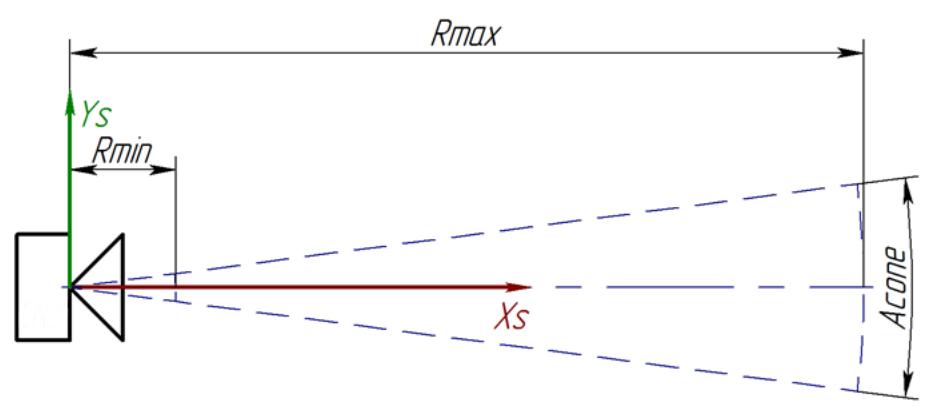

Fig. 4. Sensor coordinate system (SCS)

Using the data entered at the preparatory stage as the basis for calculations, the algorithm sequentially performs the following steps:

1. Clarifying the robot's position $\mathrm{X}_{\mathrm{rW}}, \mathrm{Y}_{\mathrm{rW}}$, and the robot's $\operatorname{Dir}_{\mathrm{sR}}$ direction in the world coordinate system (WCS) (see Fig. 3).

2. Specifying the $X_{s w}, Y_{s w}$, and $\operatorname{Dir}_{s w}$ position of each $X_{s w}, Y_{s w}$, and $\operatorname{Dir}_{s w}$ sensor in WCS.

3. The direction of the cone boundaries of the sensor Dcone $_{L}$, Dcone $_{R}$ in WCS is calculated.

4. Updating Sind sensor readings at the current time.

5. If the sensor reading is less, than the maximum range set at the preparatory stage $S_{\text {ind }}>R_{\max }$, the creating obstacle points function is called.

6. Calling the removal function, which removes points, located inside the sensor's action cone.

It is worth noting, that due to the features of the algorithm, the functions for creating and deleting points can be put in parallel execution relative to each other. In points generation function, the position of the created $\mathrm{P}_{\mathrm{w}} \mathrm{x} \mathrm{P}_{\mathrm{w}} \mathrm{y}$ points in WCS is calculated, based on the sensor reading and its position in WCS (Fig. 5).

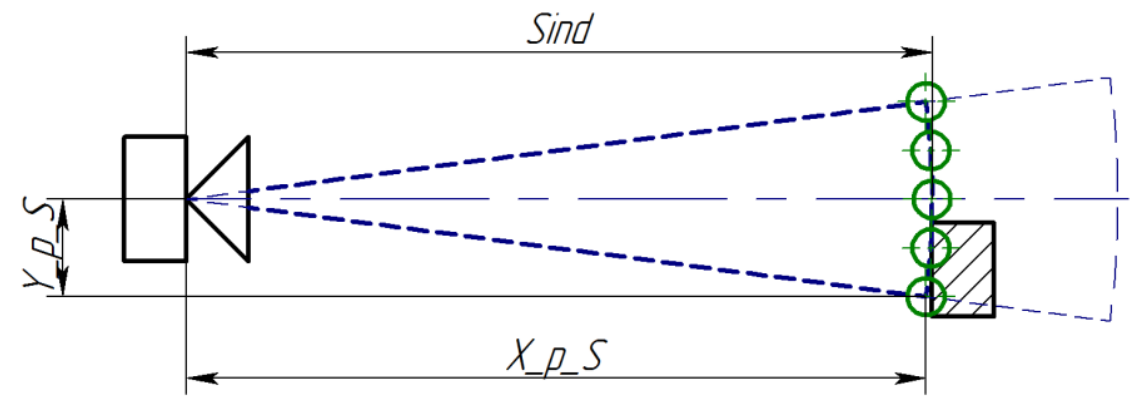

Fig. 5. Obstacles detection by points/spots generation.

The created obstacle points are evenly located on the arc formed by a sector with a radius equal to the sensor reading. To avoid creating an excessive number of obstacle points, the creation algorithm has a condition, that does not allow creating a new point within a certain radius from an existing one. Since the algorithm creates points, that are located at a significant distance from the actual obstacle (see figure 5), it is necessary to use the point removal function (Fig. 6). 


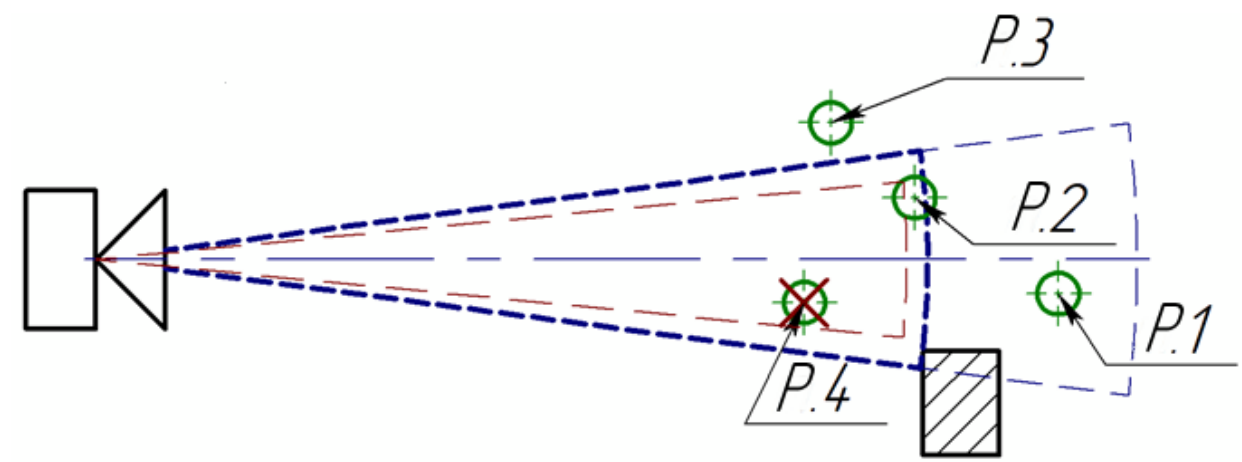

Fig. 6. Removing obstacle-points.

To optimize the algorithm, the point removal function works in several stages. At the first stage, the distance $\mathrm{R}_{\mathrm{ts}}$ from the point to the sensor is calculated. If this distance exceeds the value of the current sensor reading minus the buffer, as in points P1 and P2 from the figure above, no further checks are performed. A small buffer is used to avoid a conflict between the point removal function and the point generation function. To simplify the algorithm, this buffer can be assumed to be equal to $\mathrm{R}_{\mathrm{exc}}$. The next step is to calculate the angle of rotation (in WCS) of the straight line formed between the location of the sensor and the point. The condition (1) is checked.

$$
D_{\text {coleLW }}-D_{\text {buf }}>D_{\text {pointW }}>D_{\text {coneRW }}+D_{\text {buf }}
$$

$\mathrm{D}_{\text {coneLW }}, \mathrm{D}_{\text {coneRW }}$ - boundaries of the sensor's cone,

$D_{\text {buf }}-$ thickness of the buffer zone, inside which no points are removed.

The algorithm is able to work with a set of different sensors by combining their readings. The algorithm allows to combine readings of various sonar types, as well as other types of sensors, such as infrared rangefinders.

\section{Testings and experiments}

The described algorithm was tested in a virtual environment, that simulates the robot's movement and the operation of sonars. The width sonar cone was assumed to be $40^{\circ}$. Figure 7 shows the route that was taken during the simulation.

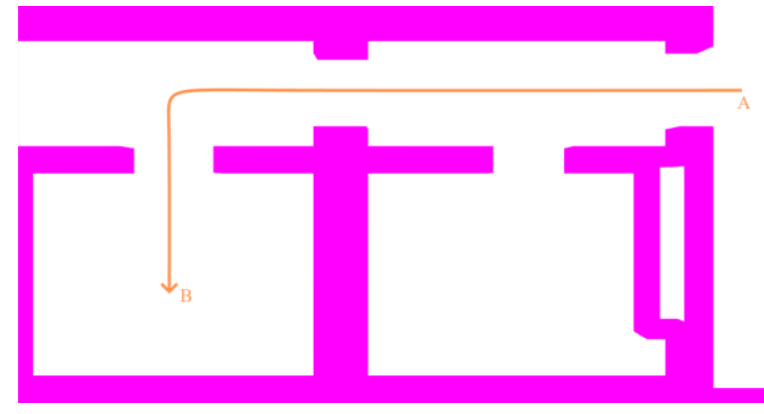

Fig. 7. The route of the robot in a virtual environment model Or the real working area.

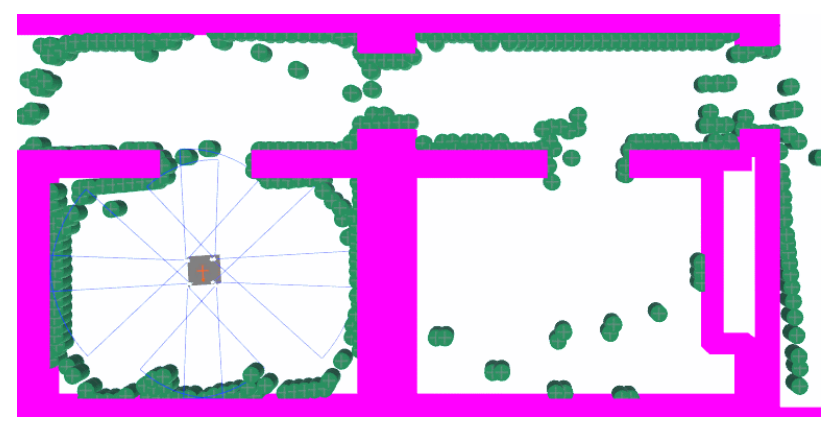

Fig. 9. The results with the beams parallel to the robot's sides

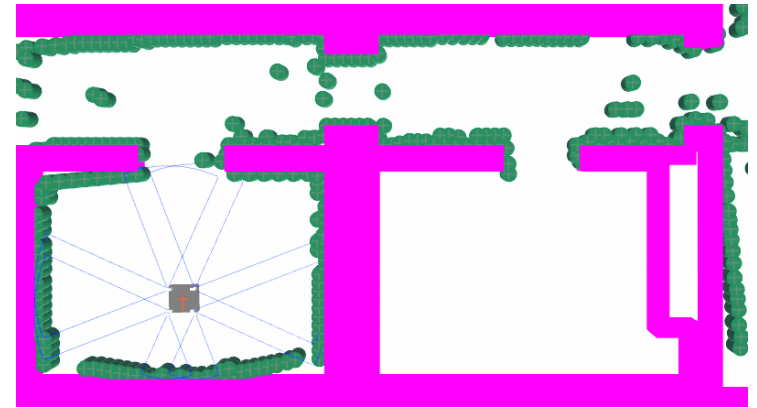

Fig. 8. The results with a perpendicular placement of the sensors

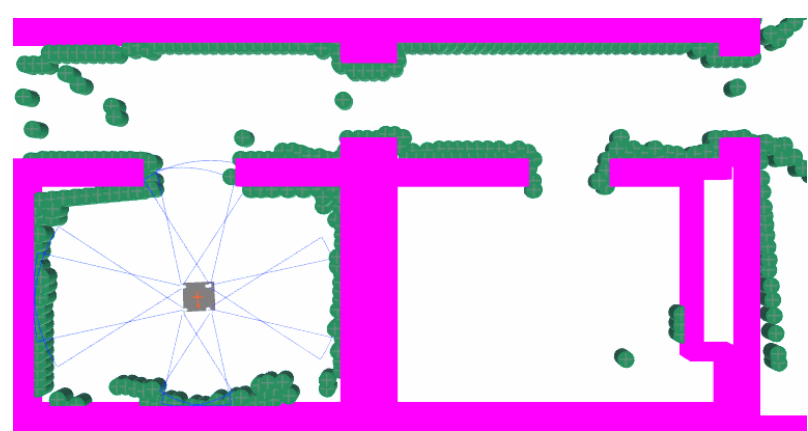

Fig. 10. The results with the convergence of the sensors' beams 
During the simulation, several options were considered for placing sonar on the robot: perpendicular to the robot body (Fig. 8), with the condition, that the beam pattern border is parallel to the robot sides (Fig. 9), with the convergence of the sensors being reduced (Fig. 10). In figures 8, 9, and 10, the sectors of the robot sensors beamare highlighted with taking into account their current reading. The points correspond to the set of points-obstacles is formed during the algorithm execution.

It is worth noting, that no additional expert rules were applied in the simulation, except for the prohibition on creating new points near deleted ones. The best results for the local mapping algorithm were found when placing sensors of 4 pairs on the sides of the robot so, that for each pair the axes of the beam pattern would be deployed at a certain angle towards each other. To confirm the functionality of the sensor layout at work, an experiment was conducted to control a virtual robot in a test environment. The operators were assigned the task to move the robot in the test environment to the target position. Several virtual test rooms of varying complexity have been prepared for this task. The operator had access to the following set of data (Fig. 11.):

1. The current coordinates of the robot as an image on a conditional map (in the robot operator window) based on odometric sensor data (their error-free operation is assumed).

2. Beam pattern cones of sonar at the current time.

3. The position of obstacle points-spots, constructed as a result of the algorithm based on data from sonars.

4. Direction to the target position.

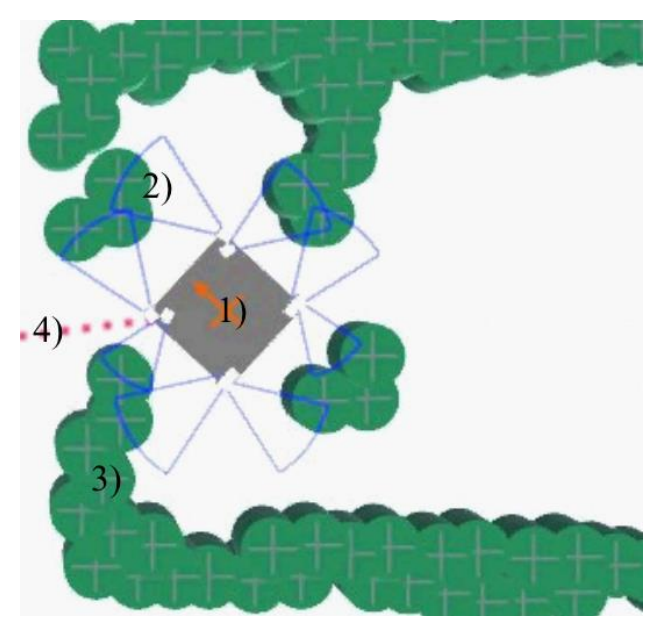

Fig. 11.Operator program view

The operators had no a-priory knowledge of the test environment. During the experiment, position of obstacles and walls of the test environment were hidden to the operator. Touching a wall or obstacle meant that the attempt is failed. To avoid operators memorizing the route, one attempt was given for each test room. An example of a test room, which includes multiple rooms, doorways, and various obstacles, is shown in figure 12.

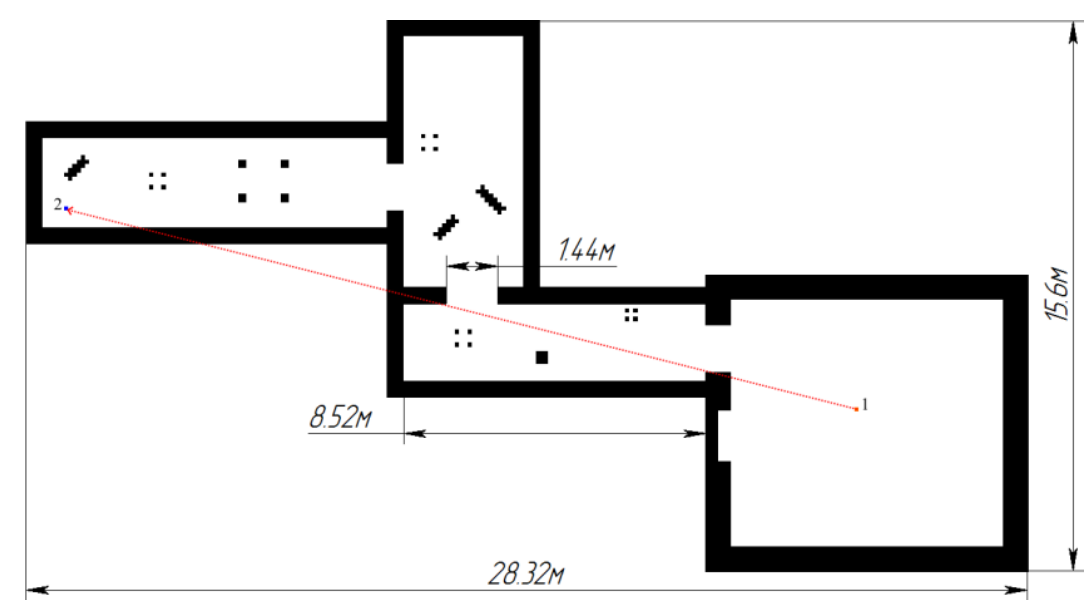

Fig. 12. The plan of the test room with the scale (model of the real rooms).

Testing has shown that operators successfully identify walls, doorways, and obstacles while controlling the virtual robot. Taking into account the absence of failures, it was concluded, that the selected concept of the sensors set on the robot is efficient. 


\section{Testing and operating HY-SRF05 sonar.}

An HY-SRF-05 sonar was proposed for conducting real life tests. This sensor has a range up to 4 meters. Operation mode (linking the mode output to ground pin) allows the HY-SRF05 to use a single pin for both trigger and echo, thus saving valuable contacts on your controller. When the mode pin is not connected, the HY-SRF05 works with separate trigger and echo pins, like the HC-SRF04, HY-SRF05 includes a small delay before the echo pulse to give slower controllers, such as Basic Stamp, time to execute their pulse in commands. HY-SRF05 can be switched on every 50 MS or 20 times every second. User should wait 50 MS until the next launch, even if the SRF05 detects a close object and the echo pulse is shorter. This is done so, that the ultrasonic "hoot" disappears and does not cause a false echo at the next measurement. The HY-SRF05 radiation pattern coincides with the beam width, which is a function of the surface area of the transducers, and is fixed. The beam pattern of the transducers used on the HY-SRF05, taken from the manufacturer's specification, is shown below (Fig.13).

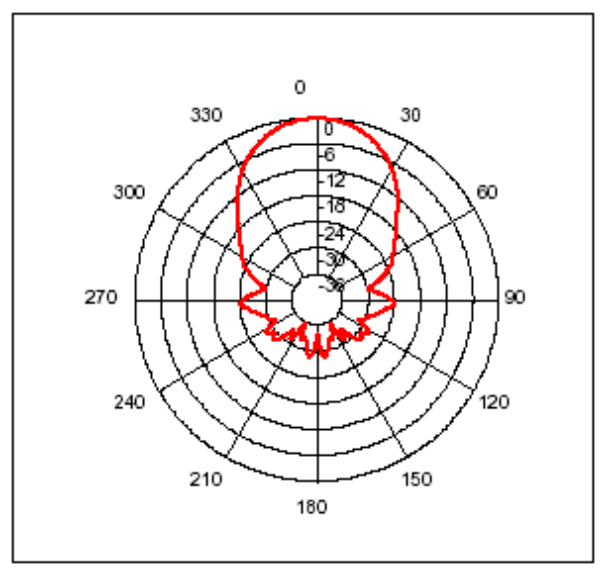

Fig. 13. Beam pattern of SRF-05

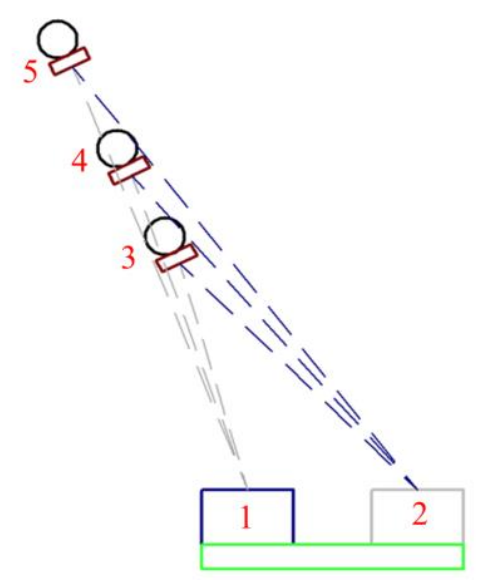

Fig. 14. Schematic representation of the experiment.

To use HY-SRF-05 sonar in our project the following problems must be solved:

- Ensure that the sensor connection to the control computer via the I2C bus. The SRF05 sensor does not operate over the I2C Protocol. Thus, there are several ways to solve this problem. One is to modify the board HY-SRF05. The other is addition of an expansion board to which all sonar will be connected and which will communicate with the main Arduino via I2C.

- Build a mathematical and computer model of the sonar, were the Python programming language is used to build the computer model.

- Determine the actual beam pattern. The beam pattern of a real sensor may differ from the data in sensor specifications.

In order to find out the real directional pattern, you need to conduct a practical experiment. The idea of the experiment is as follows: the sonar is fixed motionless, and a reflector moving around on different distanced (Fig. 14.). The lower part of the figure shows the HY-SRF05 sonar, 1 - the sonar signal trigger, and 2 - the echo receiver, respectively. The sound wave emitted by the sensor trigger is shown by the left dashes, and the sound wave reflected from the surface is shown by the right dashes. With the help of reflector, that we moved to determine the boundary points, for example, from position 3 to position 4 , or to position 5, we got a sample of points, that we used to build a real beam cone of sonar.

\begin{tabular}{|c|c|c|}
\hline Point number & X coordinates, cm & Y coordinates, cm \\
\hline 1 & 0 & 0 \\
\hline 2 & 31,5 & 20 \\
\hline 3 & 52 & 29 \\
\hline 4 & 68 & 38 \\
\hline 5 & 89,5 & 51 \\
\hline 6 & 112 & 60,5 \\
\hline 7 & 137 & 67 \\
\hline 8 & 156 & 72 \\
\hline 9 & 178 & 79,5 \\
\hline 10 & 193 & 81,5 \\
\hline 11 & 212 & 84 \\
\hline 12 & 235 & 84 \\
\hline
\end{tabular}

Table 1. The coordinates of the points from the beam pattern. 
In the second part of the experiment, the sensor was installed at a distance of $60 \mathrm{~cm}$ from the wall (Fig. 15), the sensor was rotated with a discreteness of 10 degrees.

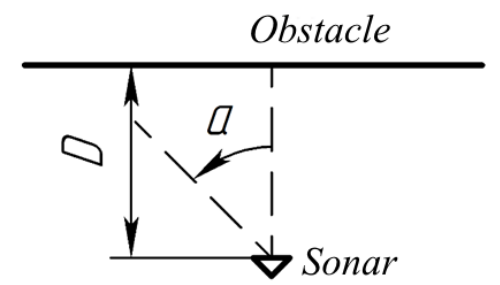

Fig. 15. Diagram of the second part of the experiment

\begin{tabular}{|c|c|}
\hline Sensor rotation angle, deg & Measured distance, $\mathbf{c m}$ \\
\hline 0 & 60 \\
\hline 10 & 60,2 \\
\hline 20 & 60,5 \\
\hline 30 & 60,9 \\
\hline 40 & 61,5 \\
\hline 50 & 178,4 \\
\hline
\end{tabular}

Table 2. The coordinates of the points for the beam pattern.

Based on the data in tables, we can conclude, that to correctly measuring distance, the sensor cant be rotated relative to the obstacle not more, than $40^{\circ}$.

\section{Combination with LIDAR}

The proposed algorithm allows combining LIDAR and sonar readings in a single algorithm. This is facilitated by the fact, that the mapping algorithm, using sonar and LIDAR, allows you to determine the position of obstacles in the form of points on the map. Combining LIDAR and sonar will increase the reliability of the system by processing the readings of different sensor types. When solving conflicts between data by different sensor types, worst-case scenario should be assumed:

- To avoid collision with transparent obstacles, points (spots), generated on the base of sonar data, should be deleted due to the information from lidar data.

- Same rules should be applied to the LIDAR based data, to avoid collision with the soft objects.

\section{Conclusion}

The proposed algorithms for creating an obstacle points (spots) for constructing (approhimating) a map of the nearest robot operating space in a previously unknown environment has very good prospects for applications, which is shown by the example of modeling in a virtual environment. But it is worth noting that the use of this algorithm as part of a real robot requires additional experiments aimed at eliminating false points-obstacles and optimizing the algorithm as a whole. Further interpretation of sensor readings and results of local mapping of the operating space formed the basis for building expert schemes for algorithms for effective control, navigation, and obstacle avoidance of mobile robots. The use of this technology to lidar data processing helps us to reduce 10-100 times the required calculations and use primitive on-boad microprocessors instead of PC-tipe processors.

\section{References}

[1] Katalinich B., Ksenzenko A. Ya., Kuvshinov S. V., Mazanov Yu. S., Rysev E. A. Pryanichnikov V. E., Helemendik R. V., Epikov S. R. The development of distributed software and hardware robotarium. EXTREME ROBOTICS. // Proceedings of the International scientific and technical conference. - Saint Petersburg: Ap4print LLC, 2016. - 480c

[2] Pryanichnikov V. E., Kuvshinov S. V., Helemendik R. V., Kharin K. V. Intelligent robotronics: scientific and educational technology and building a network of robotariums// The design of the future. Problems of digital reality: proceedings of the 1st International conference (February 8-9, 2018, Moscow). — Moscow: KIAM, 2018. — Pp. 57-64

[3] Ksenzenko, A.; Kuvshinov, S.; Poduraev, Y.; Prysev, E. \& Khelemendik, R. (2016). Intelligent Robotronics: Hardware-Software Complexes of Robotariums, Proceedings of the 27th DAAAM International Symposium, pp.0225-0229, B. Katalinic (Ed.), Published by DAAAM International, ISBN 978-3-902734-08-2, ISSN 1726-9679, Vienna, AustriaDOI: 10.2507/27th.daaam.proceedings.033 
[4] I. A. Baranov, V. I. Denisov, K. I. Kiy, K. B. Kirsanov, A. A. Kirilchenko, B. M. Levinsky, V. E. Pryanichnikov, V. G. Shagalov. Adaptive control of a crawler robot in a mobile patrol tasks. - Information-measuring and control systems, 2006 vol. 4, no. 1-3, p. 91-98

[5] V. E. Pryanichnikov. Remote sensors as part of mobile robot motion control systems. - Information-measuring and control systems, Moscow: Radio-Engineering, No. 1, 2008, p. 5-18

[6] Davydov O. I., Platonov A. K. Netpassframov - combined model of the mobile robot operating environment // Preprints of KIAM 2015. No 15. 28 p. URL: http://library.keldysh.ru/preprint.asp?id=2015-15

[7] Tardós, J. D., Neira, J., Newman, P. M., \& Leonard, J. J. (2002). Robust Mapping and Localization in Indoor Environments Using Sonar Data. The International Journal of Robotics Research, 21(4), 311-330. doi:10.1177/027836402320556340

[8] D Hutabarat, M. Rivai, D. Purwanto and H. Hutomo "Lidar-based obstacle avoidance for the autonomous mobile robot", International Conference on Information \& Communication Technology and System (ICTS), pp. 197-202, 2019.

[9] Okuda, K., Miyake, M., Takai, H., \& Tachibana, K. (2010). Obstacle arrangement detection using multichannel ultrasonic sonar for indoor mobile robots. Artificial Life and Robotics, 15(2), 229-233. doi:10.1007/s10015-0100799-2

[10] H. Gao, Q. Wei, Y. Yu, et al., Mobile robot obstacle avoidance algorithms based on information fusion of vision and sonar. Int. J. Future Gener. Commun. Netw. 9(8), 111-120 (2016) 